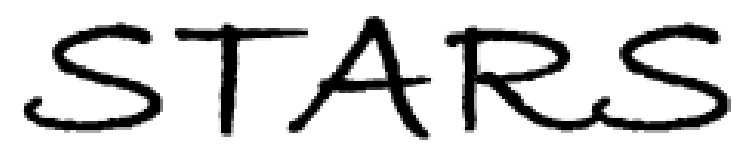

University of Central Florida

STARS

$1-1-2004$

\title{
Polarization switch using thick holographic polymer-dispersed liquid crystal grating
}

\author{
Yan-Qing Lu \\ University of Central Florida \\ Fang Du \\ University of Central Florida \\ Shin-Tson Wu \\ University of Central Florida
}

Find similar works at: https://stars.library.ucf.edu/facultybib2000

University of Central Florida Libraries http://library.ucf.edu

This Article is brought to you for free and open access by the Faculty Bibliography at STARS. It has been accepted for inclusion in Faculty Bibliography 2000 s by an authorized administrator of STARS. For more information, please contact STARS@ucf.edu.

\section{Recommended Citation}

Lu, Yan-Qing; Du, Fang; and Wu, Shin-Tson, "Polarization switch using thick holographic polymer-dispersed liquid crystal grating" (2004). Faculty Bibliography 2000s. 4563.

https://stars.library.ucf.edu/facultybib2000/4563

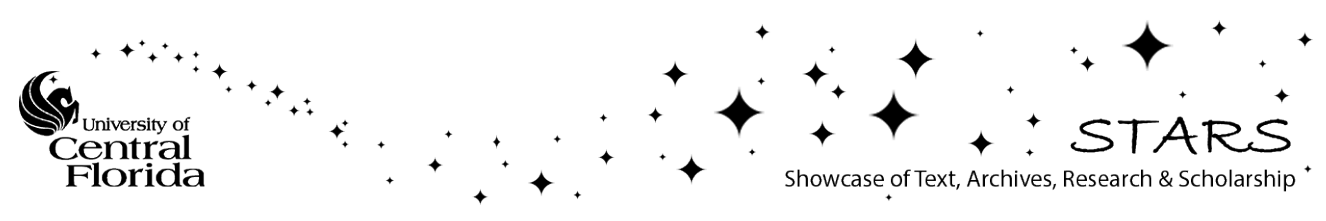




\section{Polarization switch using thick holographic polymer-dispersed liquid crystal grating}

Cite as: Journal of Applied Physics 95, 810 (2004); https://doi.org/10.1063/1.1633337

Submitted: 18 August 2003 . Accepted: 22 October 2003 . Published Online: 20 January 2004

Yan-qing Lu, Fang Du, and Shin-Tson Wu

\section{ARTICLES YOU MAY BE INTERESTED IN}

Electrically switchable volume gratings in polymer-dispersed liquid crystals Applied Physics Letters 64, 1074 (1994); https://doi.org/10.1063/1.110936

Liquid-crystal diffraction gratings using polarization holography alignment techniques Journal of Applied Physics 98, 123102 (2005); https://doi.org/10.1063/1.2146075

A low voltage submillisecond-response polymer network liquid crystal spatial light modulator Applied Physics Letters 104, 023305 (2014); https://doi.org/10.1063/1.4862474

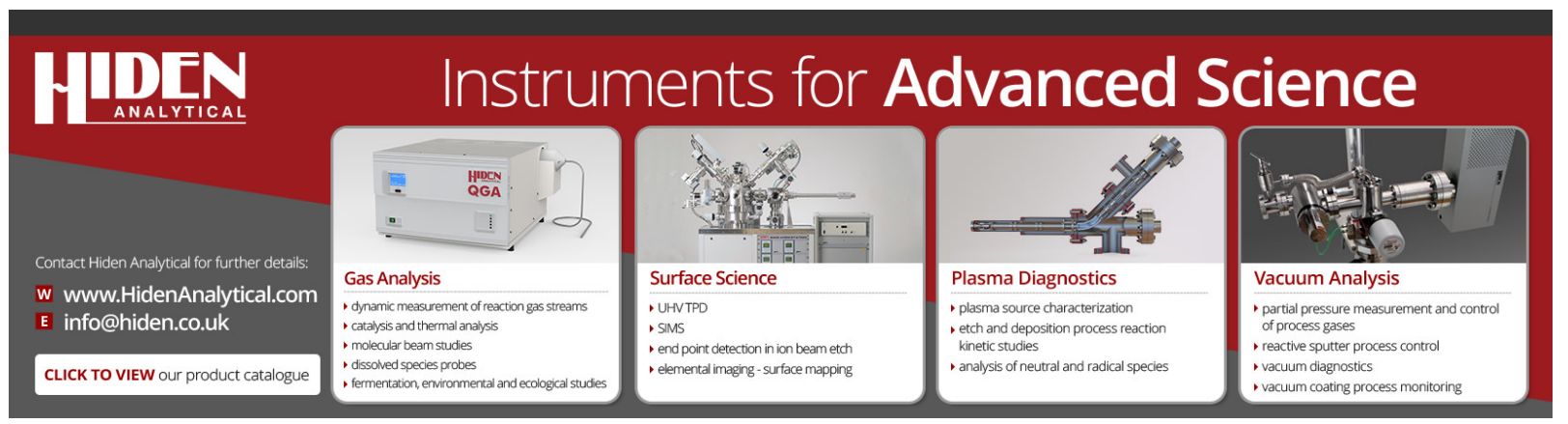




\title{
Polarization switch using thick holographic polymer-dispersed liquid crystal grating
}

\author{
Yan-qing Lu, Fang Du, and Shin-Tson Wu ${ }^{\mathrm{a})}$ \\ School of Optics/CREOL, University of Central Florida, Orlando, Florida 32816
}

(Received 18 August 2003; accepted 22 October 2003)

\begin{abstract}
Theoretical study of the optical diffraction properties of holographic polymer-dispersed liquid crystal (HPDLC)-based thick volume grating was presented. It is found that the HPDLC grating can selectively diffract the $s$-polarized light only, $p$-polarized light only, or both $s$ and $p$ beams by selecting suitable grating parameters. When an electric field is applied, the $s$ - and $p$-polarized lights exhibit different diffraction behaviors and, thus, the tunable dual-state or three-state polarization switching could be realized. (C) 2004 American Institute of Physics. [DOI: 10.1063/1.1633337]
\end{abstract}

\section{INTRODUCTION}

Holographic polymer-dispersed liquid crystal (HPDLC)based volume grating has important applications in freespace optical communications, fiber optics, integrated optics, sensor system, optical data storage, and optical computers. ${ }^{1-3}$ The HPDLC grating is a composite system of liquid crystal (LC) and polymer. If the mixture of photosensitive prepolymer and LC are exposed to a UV light with periodically varied intensity, which is normally a two-beam interference pattern, periodic phase separation could be induced. In the bright regions, polymerization occurs more rapidly than that in the dark regions. The $\mathrm{LC}$ molecules diffuse to dark regions while the monomer diffuses to the bright regions. ${ }^{4,5}$ As a consequence, a superimposed polymer density and LC droplet grating is formed with the refractive index modulated periodically.

Being different from normal volume gratings, the optical anisotropy of a LC makes the light diffraction in HPDLC grating exhibit some special characteristics. It was demonstrated that the HPDLC acts as an isotropic volume grating at monomer concentration larger than $24 \%$, where the conventional Kogelnik's diffraction theory ${ }^{6}$ could be applied. ${ }^{7}$ On the contrary, the LC droplets in HPDLC have a dominated director orientation if the monomer concentration is low. Its order parameter is close to 0.8 if the monomer concentration is less than $8 \%,^{7}$ thus the LC birefringence will affect the diffractive properties of the grating. In this case, the Kogelnik's diffraction theory is invalid and should be replaced with the two-wave coupled-wave theory of anisotropic media. ${ }^{8-10}$ To date, several theoretical and experimental works have been done on the diffraction properties of HPDLC. However, these studies mainly focused on the HPDLC with the cell gaps only around $10 \mu \mathrm{m}$. There is still not much study on the diffraction properties of thick cell gap (e.g., $100 \mu \mathrm{m})$ HPDLC gratings.

In this article, the diffraction properties of ideal thick HPDLC gratings with high monomer concentration were studied theoretically. Here, the thick grating refers to grating thickness around $100 \mu \mathrm{m}$ or thicker. The suggested monomer

\footnotetext{
${ }^{\text {a)} E l e c t r o n i c ~ m a i l: ~ s w u @ m a i l . u c f . e d u ~}$
}

concentration is larger than $70 \%$ to ensure high optical isotropy without external field. The corresponding LC concentration is thus less than $30 \%$. We define this condition as low LC concentration or high monomer concentration. In Sec. II, we present results of this thick HPDLC grating without an electric field. We show that the HPDLC grating can selectively diffract light with one or two polarization states by suitable grating design. The corresponded working conditions and system performances are investigated. An application in the fiber-optic area as a reconfigurable channel-based wavelength switch is proposed. In Section III, the diffraction properties of thick HPDLC gratings with applied field are investigated. Because of the different diffraction behaviors of $s$ - and $p$-polarized lights under an external field, the HPDLC may be used to construct a dynamic two- or three-state polarization switch that can selectively change the polarization status of the diffracted beam. Potential applications of these devices are also discussed.

\section{HOLOGRAPHIC POLYMER-DISPERSED LIQUID CRYSTAL WITHOUT AN EXTERNAL FIELD}

In a HPDLC, if the monomer concentration is high, its diffraction properties are very similar to that of a normal volume gating made with isotropic materials. ${ }^{7}$ Let us consider an unslanted grating with thickness $L$. We model it as having two different domains. Domain A is solid polymer region without any LC droplets, while domain $\mathrm{B}$ contains lower polymer content and a volume fraction of LC droplets. This model is based on the scanning electron microscope photographs published in Ref. 11. If the refractive indices in domains $\mathrm{A}$ and $\mathrm{B}$ are $n_{A}$ and $n_{B}$, respectively, $n_{B}$ can be expressed as

$$
n_{B}=f n_{\mathrm{LC}}+(1-f) n_{A},
$$

where $f$ is the filling fraction of the LC droplets, $n_{\mathrm{LC}}$ is the refractive index of LC droplets, which is the threedimensional average index of the LC. Please note that the $n_{A}$ here is not exactly the same as the index of pure polymer because there are still some LC molecules trapped in the polymer matrix and do not phase separate to form the droplets. However, because the LC in domain A are fixed and 
cannot be reoriented by the external electric field, ${ }^{12} n_{A}$ may be treated as the refractive index of the polymer without affecting the results.

According to Kogelnik's diffraction theory, ${ }^{6}$ the Bragg diffraction efficiencies for the $s$ - and $p$-polarized lights are given by

$$
\begin{aligned}
& \eta_{s}=\sin ^{2}\left(\frac{\pi n_{1} L}{\lambda \cos \theta}\right), \\
& \eta_{p}=\sin ^{2}\left[\frac{\pi n_{1} L \cos (2 \theta)}{\lambda \cos \theta}\right],
\end{aligned}
$$

where $L$ is the thickness of the HPDLC, $\theta$ is the Bragg angle within the grating, and $n_{1}$ is the first Fourier component of the refractive index modulation. It can be expressed as $n_{1}$ $=\left|n_{A}-n_{B}\right| / 2$, where a sinusoidal index profile is assumed. ${ }^{7,8}$

In the isotropic HPDLC, the refractive index modulation is identical for the $s$ and $p$ beams. The only factor that determines the different diffraction behaviors between different polarization states is the $\cos (2 \theta)$ term in Eq. (3), which originates from the unparallel electric fields between the incident $p$ beam and the diffracted $p$ beam. This explains why the $p$-polarized light normally has a lower diffraction efficiency than that of the $s$-polarized light. This phenomenon has been used as a criterion to judge whether a HPDLC grating is isotropic or anisotropic. However, in the case of a thick HPDLC grating, there exists periodic energy exchange between the undeflected zeroth-order beam and the first-order diffracted beam. The diffraction efficiency of the $p$-polarized light may exceed that of the $s$-polarized light because they do not evolve synchronously. Some useful characteristics thus are obtained.

When $|\cos (2 \theta)|=(2 \ell-1) /(2 k-1)=1 / 3,1 / 5,3 / 5, \ldots$, where $k$ and $\ell$ are both positive integers and $k>\ell$, it is possible to make $\eta_{p}=\eta_{s}=1$ for a specific grating thickness, which means that the $p$ - and $s$-polarized light can reach their maxima simultaneously. This is a very attractive feature. As we know, typical bulk gratings including the surface relief gratings and volume gratings are normally polarization dependent. The unequal efficiencies for different polarization states result in polarization dependent loss (PDL) in related devices. Normally, the PDL has severe negative impact on the performance of an instrument. For example, in the fiberoptic communication system, PDL induces a loss variation in the transmitted signal and then degrades the optical signalto-noise ratio. However, if a polarization independent HPDLC grating is employed, the intrinsic PDL could be eliminated so that it is unnecessary to use the complicated optical elements to suppress the PDL. Thus, the optical system becomes simpler and more economic.

Figure 1 shows the simulation result of a HPDLC grating with $\cos (2 \theta)=1 / 3$, i.e., $k=2$ and $\ell=1$. The corresponding Bragg angle in the grating is $35.26^{\circ}$. If the average refractive index of the HPDLC is 1.5 , the grating density is calculated to be 1118 grooves $/ \mathrm{mm}$ for working in the 1.55 $\mu \mathrm{m}$ telecommunication wavelength window. From Fig. 1, both $s$ - and $p$-polarized lights can reach their maximal efficiencies if the product of index modulation and grating thickness is $1.9 \mu \mathrm{m}$.

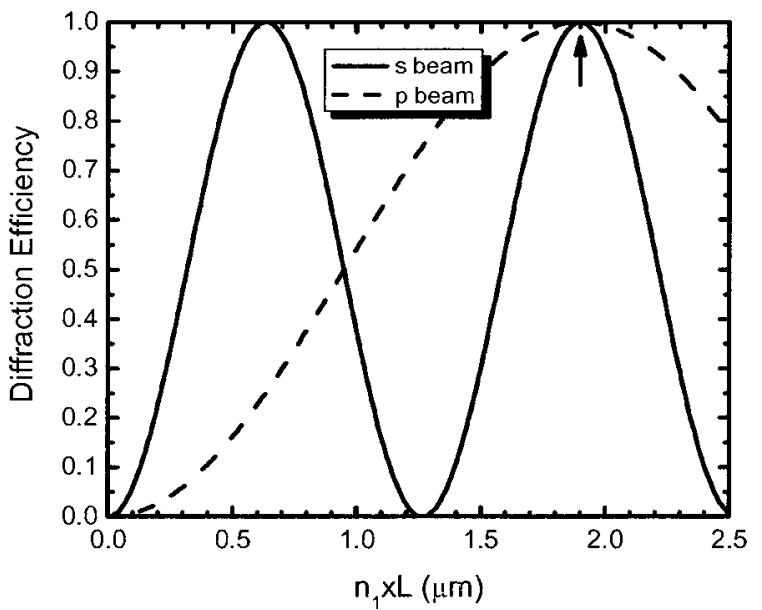

FIG. 1. Diffraction efficiency of a HPDLC grating as a function of the product of refractive index modulation and grating thickness. The solid line represents the $s$-polarized light and the dashed line is for the $p$-polarized light. The diffraction efficiency for both polarization states can reach their maximum simultaneously in this case.

For many practical applications, the gating has to work in a spectral band rather than a specific laser line. The spectral response of the HPDLC grating is studied. Results are displayed in Fig. 2. Figure 2 shows that the grating has very flat response across the whole $C$ band defined by the International Telecommunication Union (ITU). The maximum PDL in this band is less than $0.02 \mathrm{~dB}$, which is negligible.

Besides diffracting both polarization states, the HPDLC grating is also capable of diffracting only one of them. When $|\cos (2 \theta)|=(2 \ell-1) / 2 k=1 / 2,1 / 4,3 / 4, \ldots$, the diffraction efficiency of the $p$ wave can reach its maximum, while there is no diffraction for the $s$ wave, i.e., $\eta_{p}=1, \eta_{s}=0$. This is a very interesting result, which means that the HPDLC grating acts as a polarization beam splitter while maintaining its diffractive ability. An example is shown in Fig. 3(a) with $\cos (2 \theta)=1 / 2$. The work condition for this case is $n_{1} \times \ell$ $=1.34 \mu \mathrm{m}$, which can be satisfied by the suitable design of the grating thickness and accurate control of index modulation. Similarly, the HPDLC grating may also be designed to only diffract the $s$ polarization while keeping high transmission for the $p$ polarization. The general condition for this

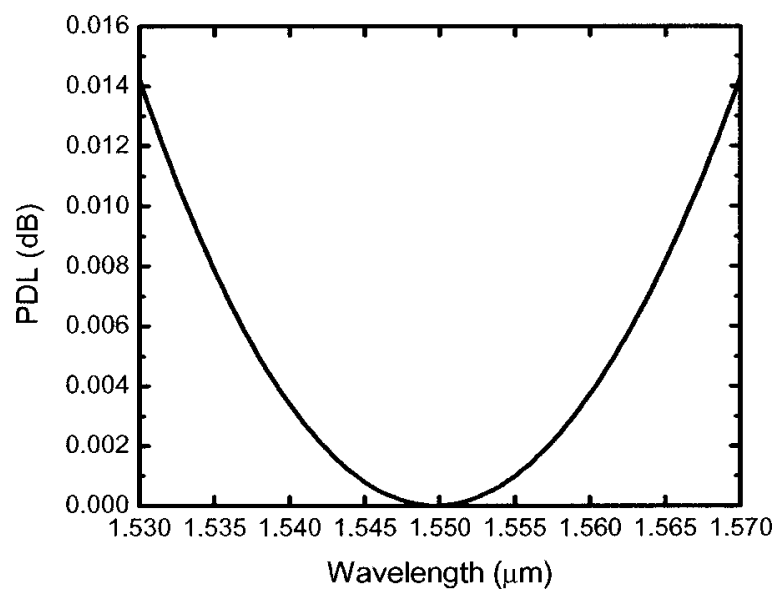

FIG. 2. The PDL of a HPDLC grating within the ITU $C$ band. 

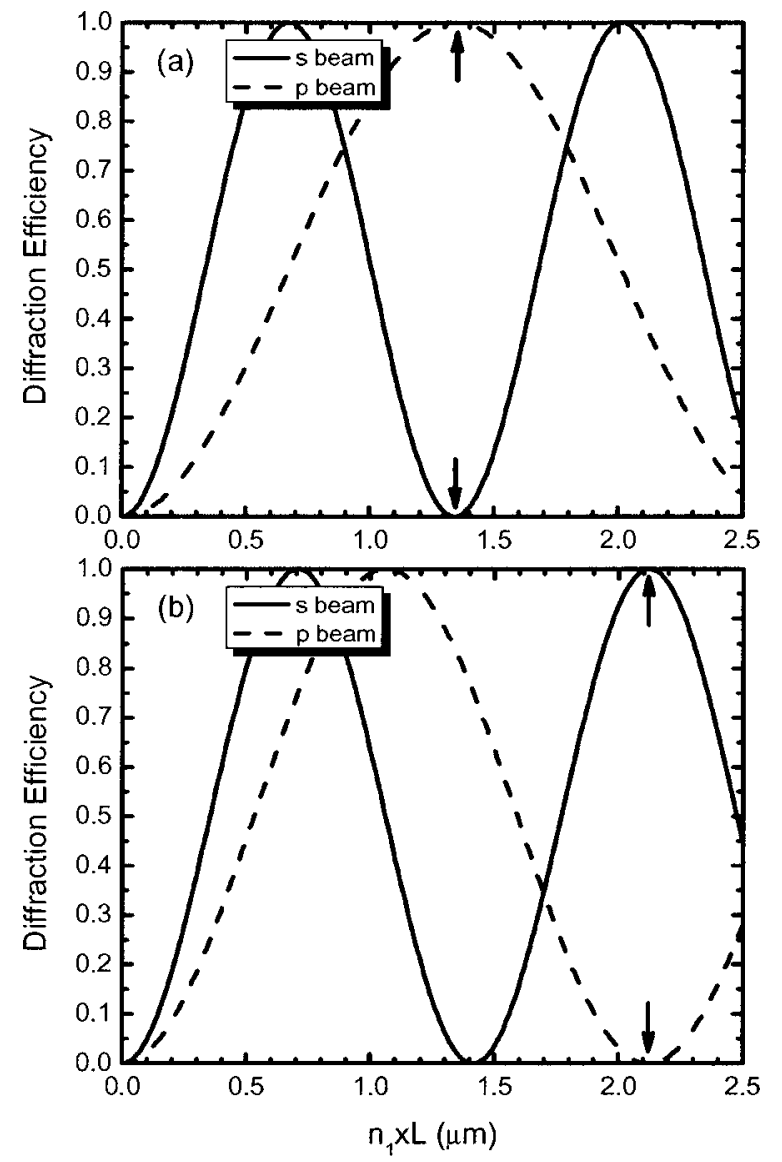

FIG. 3. Diffraction efficiency of HPDLC grating as a function of the product of refractive index modulation and grating thickness when (a) $\cos (2 \theta)$ $=1 / 2$ and $(\mathrm{b}) \cos (2 \theta)=2 / 3 . \theta$ is the Bragg angle in the HPDLC. The solid line represents the $s$-polarized light and the dashed line is for the $p$-polarized light.

feature is $|\cos (2 \theta)|=2 \ell /(2 k+1)=2 / 3,2 / 5,4 / 5, \ldots$ Figure $3(\mathrm{~b})$ shows the diffraction efficiency as a function of $n_{1} \times \ell$ for both polarization states when $\cos (2 \theta)=2 / 3$. As $n_{1} \times \ell$ $=2.12 \mu \mathrm{m}$, only the $s$ polarization is diffracted and the $p$ polarization passes though the grating directly without diffraction.

To evaluate the performances of these HPDLC-gratingbased polarization beam splitters, the contrast ratio (defined as the ratio between the light intensity of two polarization states) is the main factor. Figure 4 shows the calculated contrast ratio within the ITU $C$ band for a HPDLC-based beam splitter as described above. The system parameters are the same as those used in Fig. 3(a). If the parameters for Fig. 3(b) are used, results remain unchanged. From Fig. 4, the contrast ratio is over $30 \mathrm{~dB}$ for the most part of the $C$ band. This contrast ratio is acceptable for many telecommunication applications. If a higher contrast ratio is needed, cascading HPDLC layers may be a viable approach.

The polarization splitting properties of the HPDLC grating have many applications, for example, optical addressing, variable optical attenuating, beam steering, etc. However, because the HPDLC grating combines the wavelength diffraction property and polarization splitting ability together, it will enable other applications. Figure 5 illustrates a possible ap-

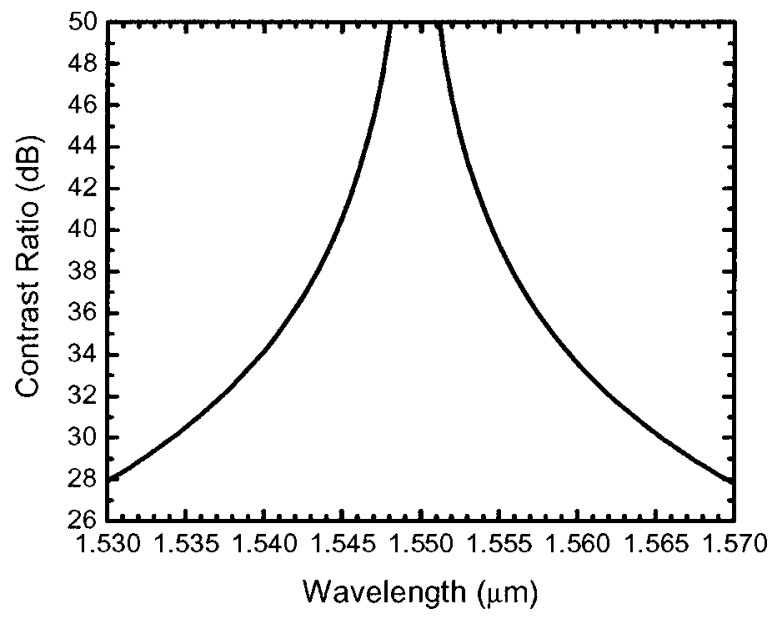

FIG. 4. Calculated contrast ratio of a HPDLC grating working as a polarization beam splitter. The contrast ratio is defined as the absolute value of the efficiency ratio between two polarization states.

plication as a reconfigurable switch in WDM communication systems. The input polarized beam (for example, $s$ polarization), that contains plenty of frequency components, is diffracted by the first HPDLC grating. An entrance converging lens focuses the frequency-divided components upon a polarization modulator array, such as $90^{\circ}$ aligned twisted nematic (TN) LC or Faraday rotator that can control the polarization statuses of the output beams. Another identical lens and grating that act reciprocally to recollimate and recombine the frequency-separated beams into an optical fiber. Because each unit in the modulator array can be controlled individually, we can intentionally change the polarization state of any channel. If the polarization of a specific channel is modified, the corresponding beam is not diffracted back to the output port. A channel-based wavelength blocker or switch is thus obtained. Furthermore, it is not difficult to modify this system to be an optical channel power equalizer or even a reconfigurable optical add-and-drop multiplexer. All of these devices have important applications in the WDM fiber-optic communication systems, either for long-haul or for metropolitan networks.

All of our discussions so far are based on Kogelnik's diffraction theory, thus, our results should apply to other normal isotropic volume gratings. However, the fundamental difference between the HPDLC and the normal volume gating is its tunability. The related optical properties and possible applications will be discussed in Sec. III.

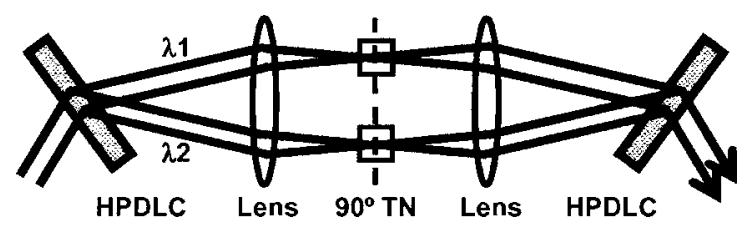

FIG. 5. System schematic diagram of a channel-based reconfigurable wavelength switch by using HPDLC gratings for both frequency diffraction and polarization beam separation. This wavelength switch may be used in the WDM fiber-optic communication systems. 


\section{DIFFRACTION UNDER AN EXTERNAL FIELD}

When a sufficiently high voltage is applied to a HPDLC cell, the polymer portion in the material remains unchanged while the LC droplets are reoriented. When a HPDLC is in the field-off state, the orientation of the droplets is random through the sample. When the HPDLC is in the field-on state, the LC directors inside the droplets are aligned along the electric-field direction. Under such a circumstance, the normal incident light encounters the same refractive index for different polarization states. However, the incident light should always propagate obliquely in a HPDLC grating to satisfy the Bragg condition. If the polarization of the incident light is perpendicular to the plane defined by the LC director and the wave vector of the incident light, this $s$ polarization encounters the ordinary refractive index $n_{o}$ in the LC droplets. If the polarization of the incident light is in the plane defined by the LC director and the wave vector, which is the case of $p$ polarization, the refractive index in the LC droplets is different. It is determined by main refractive indices of the LC, $n_{o}, n_{e}$, and the angle between the LC director and the incidence direction as

$$
n_{\mathrm{LC}, e}(\theta)=\frac{n_{o} n_{e}}{\left(n_{o}^{2} \sin ^{2} \theta+n_{e}^{2} \cos ^{2} \theta\right)^{1 / 2}},
$$

where $\theta$ is the same as the Bragg angle if the applied field is high enough to reorient the $\mathrm{LC}$ directors to be normal to the LC cell. ${ }^{13}$ The overall refractive index of the HPDLC then can be obtained by using $n_{o}$ or $n_{\mathrm{LC}, e}(\theta)$ to replace $n_{\mathrm{LC}}$ in Eq. (1). As a consequence, light with different polarizations will see the different index modulation $n_{1}$. The HPDLC grating is no longer an isotropic volume grating although it still contains high monomer concentration. Furthermore, because the orientation of the LC director is determined by the field intensity, the index modulation, and then the light diffraction should also change with the applied field. The electronically tunable HPDLC grating is thus achieved.

To study the light diffraction in anisotropic volume grating, Montemezzani and Zgonik ${ }^{10}$ extended Kogelnik's theory by two aspects. The birefringence of the refractive index modulation is taken into account by using tensor instead of scalar for relative permittivity in the solutions of coupledwave equations. Moreover, they also considered the different directions between the energy stream propagation and the wave vector, which is due to the average birefringence in the HPDLC. This phenomenon is very evident if the volume grating is recorded in a birefringent crystal. However, in the HPDLC gratings that we are studying, the intrinsic average birefringence is relatively small because of the high monomer concentration. We only need to consider the influence on the diffraction induced by the birefringence of the refractive index modulation. The diffraction efficiencies of $s$ - and $p$-polarized beams can thus be obtained by direct modification from Kogelnik's results as

$$
\begin{aligned}
& \eta_{s}=\sin ^{2}\left(\frac{\pi n_{1 o} L}{\lambda \cos \theta}\right), \\
& \eta_{p}=\sin ^{2}\left[\frac{\pi n_{1 e} L \cos (2 \theta)}{\lambda \cos \theta}\right],
\end{aligned}
$$

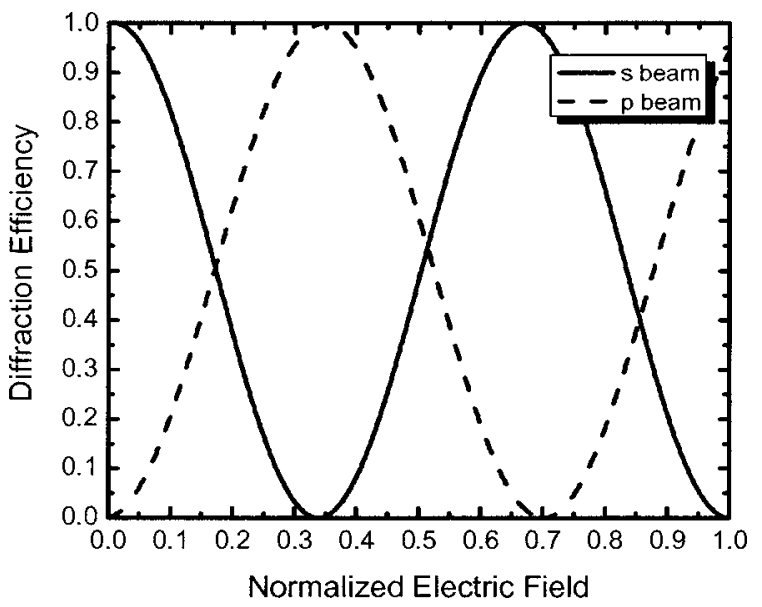

FIG. 6. Diffraction efficiency of a HPDLC grating as a function of the normalized applied electric field. The solid line represents the $s$-polarized light and the dashed line is for the $p$-polarized light.

where $n_{1 o}$ and $n_{1 e}$ represent the index modulation for $s$ - and $p$-polarized light, respectively. Although this mathematic treatment is not suitable for the high birefringent HPDLC, it is adequate for our present study. And, it is more straightforward than Montemezzani and Zgonik's method ${ }^{10}$ although the underlying physics is the same.

Figure 6 shows the calculated diffraction efficiency in a telecommunication wavelength window as a function of the normalized electric field $E_{\text {norm }}$ on the HPDLC. $E_{\text {norm }}$ is defined as

$$
E_{\text {norm }}=\left\{\begin{array}{cc}
0 & \left(E \leqslant E_{\mathrm{th}}\right) \\
\left(E-E_{\mathrm{th}}\right) /\left(E_{\mathrm{sa}}-E_{\mathrm{th}}\right) & \left(E_{\mathrm{th}}<E<E_{\mathrm{sa}}\right), \\
1 & \left(E \geqslant E_{\mathrm{sa}}\right)
\end{array}\right.
$$

where $E_{\mathrm{th}}$ and $E_{\mathrm{sa}}$ are the threshold field and saturated field, respectively. From this definition, if $E_{\text {norm }}=0$, it means that the applied field is weaker than the threshold field of the HPDLC so that directors in the LC droplets remain random. If $E_{\text {norm }}=1$, it means that a saturated field is applied and all LC droplets are reoriented along the normal of the LC cell. The systems parameters are $n_{A}=1.5, n_{o}=1.5, n_{e}=1.75, \theta$ $=65.91^{\circ}, L=92 \mu \mathrm{m}$. Assuming the LC filing factor in domain $\mathrm{B}$ is $25 \%$, thus, the original refractive index modulation is $n_{1} \approx 0.01$ for either $s$ beam or $p$ beam. When a field is applied, the refractive indices of the different polarization states undergo different changes in this special case. The index of the $s$ beam should decrease gradually and then reach zero in the end with a saturated field, because the refractive index in LC droplets matches well with that of the surrounding polymer matrix. On the contrary, because of the high birefringence (0.25) and large Bragg angle, the refractive index of the $p$ beam in LC droplets, $n_{\mathrm{LC}, e}(\theta)$, may be larger than the three-dimensional average index of randomly oriented LC droplets if an external field is applied. The index modulation for the $p$-polarized light thus increases with the field intensity, which gives rise to some unique diffraction characteristics as shown in Fig. 6. When no electric field is applied, the HPDLC acts as an isotropic grating. Due to the well-selected Bragg angle and HPDLC thickness, the 


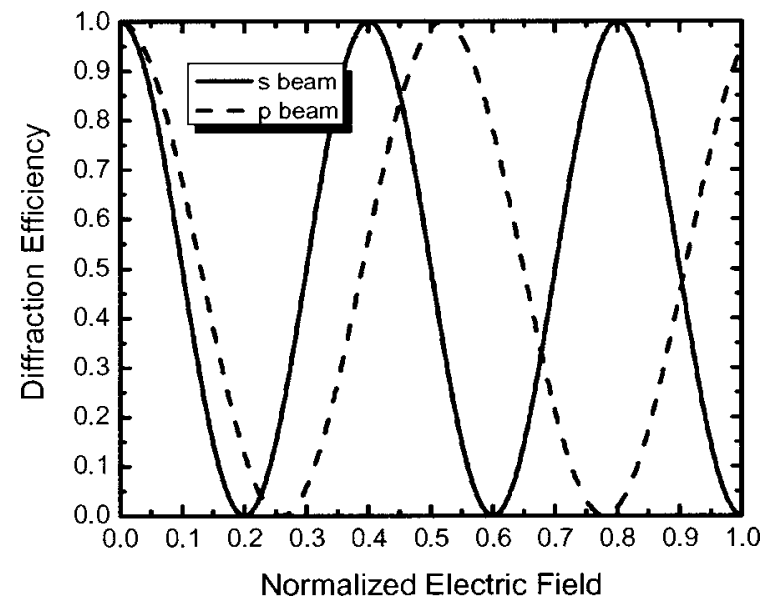

FIG. 7. Diffraction efficiency of a HPDLC grating as a function of the normalized applied electric field. The solid line represents the $s$-polarized light and the dashed line is for the $p$-polarized light. Three-state polarization switching is realized by tuning the intensity of the applied field.

$s$-polarized light is designed to be highly diffracted, whereas the $p$-polarized light has no diffraction under this condition. When an external field is applied on the sample, the index modulation for both $s$ and $p$ beams changes so that their diffraction efficiency cannot keep their original values any more. The efficiency of the $p$ beam gradually goes up from zero, while the efficiency of the $s$ beam diminishes from 1.0. For simplifying the derivation, we ideally linearize the relationship between the index modulation and the normalized field. When the normalized field reaches 0.34 , the $p$-polarized light is highly diffracted while the efficiency of $s$-state light is zero. When the electric field is 0.7 , the diffraction properties of the HPDLC grating change again with $\eta_{s} \approx 98 \%$ and $\eta_{p}=0$. The efficiency changes of $s$ and $p$ beams experience one and a half circles in the whole field span and almost switch symmetrically due to our HPDLC design: The Bragg angle, initial index modulation, and sample thickness. Obviously, a thicker HPDLC could be designed to obtain more circles of efficiency switching between the two polarization states, but it makes the sample fabrication more difficult. Thus, this is not a preferred approach.

These diffraction changes in HPDLC grating are so interesting and useful that the HPDLC works like a light filter or modulator. We may intentionally let either kind of polarization be diffracted or blocked through electric tuning. If the input light is linearly polarized, a variable optical attenuator/ modulator could be constructed without the rear polarization analyzer in common polarization modulators. ${ }^{14}$ If the input light is unpolarized, a dual-state polarization switch is thus obtained. Its physical mechanism is totally different from the ordinary TN LC and Faraday modulator, although the output polarization state is also controlled by the applied field.

In addition to the dual-state polarization switching, the three-state switching is also attainable by selecting suitable grating parameters. Figure 7 shows the calculation results. The studied grating is made with the same HPDLC for Fig. 6 but the internal Bragg angle and grating thickness are different. During simulations, $\theta=63.43^{\circ}$ and $L=166.6 \mu \mathrm{m}$ are used. From Fig. 7, the HPDLC can work in three different states by simply tuning the electric field. When $E_{\text {norm }}$ is zero, both $s$ and $p$ beams are highly diffracted; when the normalized field is 0.6 and $0.78, p$ - and $s$-only diffraction are obtained with high efficiencies, respectively. We believe that this kind of three-state polarization switch is not only fundamentally interesting but also practically useful.

The PDL and contrast ratio of these switching states are also studied. They are all close to the results shown in Figs. 2 and 4, thus no separated figures are supplied. HPDLC grating would exhibit other features by changing the grating designs. Combining the special polarization properties with light diffraction ability, other useful optical devices can also be constructed.

As for the practicality of our proposed devices, a possible concern is the angular acceptance width of the incident light because of the thick grating. For a $100 \mu \mathrm{m}$ thick HPDLC, the corresponding full width at half maximum of the angular sensitivity is $\sim 0.01$ radian, which is easily achievable for a laser beam. For a telecommunication fiber-optic device, a suitable collimator may also be used to suppress the fiber divergence angle within the acceptance band. Another issue associated with the thick grating is the device driving voltage. Normally, the field for driving the HPDLC is $\sim 10 \mathrm{~V} / \mu \mathrm{m},{ }^{15}$ thus the operating voltage for our devices will reach $\sim 1 \mathrm{kV}$ which is quite high. Although choosing suitable surfactant may reduce the HPDLC threshold and saturated field by an order of magnitude, ${ }^{16}$ this technology has not been widely used due to the problems associated with the HPDLC surfactant. Moreover, to fabricate a thick HPDLC sample is a challenging task.

\section{CONCLUSIONS}

We have investigated the light diffraction properties in HPDLC gratings for both field-off and field-on states. If no electric field is applied, the HPDLC grating with a high monomer concentration acts as an isotropic grating. The HPDLC grating could exhibit a very low PDL or high contrast ratio between different polarization states by optimizing the grating design. The corresponding required conditions for different working statuses are derived. A possible application as a smart channel-based reconfigurable switch is proposed. In a sufficiently high voltage, the HPDLC grating loses its isotropy. The $s$ - and $p$-polarized light show different diffraction characteristics with the change of field intensity. Based on this property, a dual-state or three-state special polarization switch is realized. In general, The HPDLC is such a versatile device that combines the grating diffraction, selective polarization switching, and electric field tunability. Potential applications for telecommunication light switches and variable optical attenuators are foreseeable.

\section{ACKNOWLEDGMENTS}

The authors would like to thank Charles Wong of Chorum Technologies and Hongwen Ren of UCF for useful discussions. This work is supported by AFOSR under Contract No. F49620-01-1-0377. 
${ }^{1}$ J. J. Butler and M. S. Malcuit, Opt. Lett. 25, 420 (2000).

${ }^{2}$ M. Jazbinsek, I. D. Olenik, M. Zgonik, A. K. Fontecchio, and G. P. Crawford, J. Appl. Phys. 90, 3831 (2001).

${ }^{3}$ R. L. Sutherland, V. P. Tondiglia, L. V. Natarajan, T. J. Bunning, and W. W. Adams, Appl. Phys. Lett. 64, 1074 (1994).

${ }^{4}$ C. C. Bowley and G. P. Crawford, Appl. Phys. Lett. 76, 2235 (2000).

${ }^{5}$ H. Ren and S. T. Wu, Appl. Phys. Lett. 81, 3537 (2002).

${ }^{6}$ H. Kogelnik, Bell Syst. Tech. J. 48, 2909 (1969).

${ }^{7}$ M. E. Holmes and M. S. Malcuit, Phys. Rev. E 65, 066603 (2002).

${ }^{8}$ J. J. Butler, M. S. Malcuit, and M. A. Rodriguez, J. Opt. Soc. Am. B 19, 183 (2002).

${ }^{9}$ R. L. Sutherland, J. Opt. Soc. Am. B 19, 2995 (2002).

${ }^{10} \mathrm{G}$. Montemezzani and M. Zgonik, Phys. Rev. E 55, 1035 (1997).
${ }^{11}$ T. J. Bunning, L. V. Natarajan, V. P. Tondiglia, G. Dongherty, and R. L. Sutherland, J. Polym. Sci., Part B: Polym. Phys. 35, 2825 (1997).

${ }^{12}$ P. S. Drzaic, Liquid Crystal Dispersions (World Scientific, Singapore, 1995).

${ }^{13}$ S. T. Wu and D. K. Yang, Reflective Liquid Crystal Displays, (Wiley, New York, 2001), Chap. 7.

${ }^{14}$ I. C. Khoo and S. T. Wu, Optics and Nonlinear Optics of Liquid Crystals, (World Scientific, Singapore, 1993), Chap. 2.

${ }^{15}$ V. P. Tondiglia, L. V. Natarajan, R. M. Neal, R. L. Sutherland, and T. J. Bunning, Mater. Res. Soc. Symp. Proc. 479, 235 (1997).

${ }^{16}$ R. L. Sutherland, L. V. Natarajan, V. P. Tondiglia, S. Chandra, C. K. Shepherd, D. M. Brandelik, S. A. Siwecki, and T. J. Bunning, J. Opt. Soc. Am. B 19, 3004 (2002). 